\title{
Finite groups with a trivial Chermak-Delgado subgroup
}

\author{
Ryan McCulloch \\ Communicated by Andrea Lucchini
}

\begin{abstract}
The Chermak-Delgado lattice of a finite group is a modular, self-dual sublattice of the lattice of subgroups of $G$. The least element of the Chermak-Delgado lattice of $G$ is known as the Chermak-Delgado subgroup of $G$. This paper concerns groups with a trivial Chermak-Delgado subgroup. We prove that if the Chermak-Delgado lattice of such a group is lattice isomorphic to a Cartesian product of lattices, then the group splits as a direct product, with the Chermak-Delgado lattice of each direct factor being lattice isomorphic to one of the lattices in the Cartesian product. We establish many properties of such groups and properties of subgroups in the Chermak-Delgado lattice. We define a CD-minimal group to be an indecomposable group with a trivial Chermak-Delgado subgroup. We establish lattice theoretic properties of Chermak-Delgado lattices of CD-minimal groups. We prove an extension theorem for CD-minimal groups, and use the theorem to produce twelve examples of CD-minimal groups, each having different $\mathrm{CD}$ lattices. Curiously, quasi-antichain $p$-group lattices play a major role in the author's constructions.
\end{abstract}

\section{Indecomposability and CD-minimal groups}

Let $G$ be a finite group and let $H \leq G$. Then $m_{G}(H)=|H|\left|C_{G}(H)\right|$ is the Chermak-Delgado measure of $H$ in $G$. Let $m^{*}(G)=\max \left\{m_{G}(H): H \leq G\right\}$ and then define $\mathscr{C D}(G)=\left\{H \leq G: m_{G}(H)=m^{*}(G)\right\}$. The subgroup collection $\mathcal{C D}(G)$ forms a sublattice of the lattice of subgroups of $G$. Furthermore, if $H, K \in \mathscr{C D}(G)$ and $\sigma \in \operatorname{Aut}(G)$, then we have $H^{\sigma} \in \mathscr{C D}(G),\langle H, K\rangle=H K$, $C_{G}(H) \in \mathcal{C D}(G), C_{G}(H \cap K)=C_{G}(H) C_{G}(K)$, and $C_{G}\left(C_{G}(H)\right)=H$. The least element, $T$, of $\mathcal{C D}(G)$ is known as the Chermak-Delgado subgroup of $G$, and $T$ is a characteristic, abelian subgroup of $G$ which contains the center of $G$.

This modular, self-dual sublattice of the lattice of subgroups of $G$ was first introduced in [7]. Proofs of the properties of $\mathcal{C D}(G)$ stated in the previous paragraph are found in [10, Section 1.G].

We lay down some scaffolding that is useful in proving results about the Chermak-Delgado lattice. Proposition 1 below is adapted from [1, proof of Lemma 2.4.1]. 
Proposition 1. Suppose $H, K \leq G$, and $H H^{x}=H^{x} H$ for all $x \in K$. If $K \leq H^{K}$, then $K \leq H$.

Proof. Given $x, y \in K, H^{x} H^{y}$, as $x$-conjugate of the subgroup $H H^{y x^{-1}}$, is a subgroup, and so $H^{x} H^{y}=H^{y} H^{x}$. If $H$ is normalized by $K$, the result is trivially true. So suppose there exists $x \in K$ such that $H \neq H^{x}$. By the initial remark, $H H^{x}$ satisfies the hypotheses of the proposition (instead of $H$ ). Therefore, by induction on $\left|H^{K}: H\right|$, we can assume that $K \leq H H^{x}$. Then $x=h_{1} h_{2}{ }^{x}$ for $h_{1}, h_{2} \in H$ which implies $x \in H$, a contradiction.

Corollary 1. Suppose $H, K \leq G$, and $H H^{x}=H^{x} H$ for all $x \in K$. If $H<K$, then $H^{K}<K$.

Proof. If $H<K$, then $H^{K} \leq K$. Now $H<K$ implies that $K \not L H$ so by the contrapositive of Proposition $1, K \not H^{K}$, and so in particular $H^{K} \neq K$. It follows that $H^{K}<K$.

If $H \in \mathscr{C} \mathscr{D}(G)$, then any $x \in G$ induces an automorphism of $G$, and thus $H^{x} \in \mathscr{C} \mathscr{D}(G)$, and so $\left\langle H, H^{x}\right\rangle=H H^{x}$, i.e. $H H^{x}=H^{x} H$. Thus Proposition 1 and Corollary 1 apply for any subgroup $H \in \mathscr{C} \mathscr{D}(G)$ and any subgroup $K$ of $G$.

Given $H \leq G$ and $X \subseteq G$, we use $\operatorname{core}_{X}(H)$ to denote intersection of all of the conjugates of $H$ in $X$. We note that if $H \in \mathcal{C} \mathscr{D}(G)$ and $\emptyset \neq X \subseteq G$, then $H^{X}$ and $\operatorname{core}_{X}(H)$ are both in $\mathcal{C D}(G)$.

Proposition 2. The following statements hold:

(1) If $H \in \mathscr{C D}(G)$ with $H<G$, then $H^{G}<G$.

(2) If $K \in \mathcal{C D}(G)$ with $Z(G)<K$, then $Z(G)<\operatorname{core}_{G}(K)$.

Proof. Part (1) follows directly from Corollary 1.

If $Z(G)<K$, then $C_{G}(K)<G$, so by Corollary $1, C_{G}(K)^{G}<G$. And so $Z(G)<C_{G}\left(C_{G}(K)^{G}\right)=\operatorname{core}_{G}\left(C_{G}\left(C_{G}(K)\right)=\operatorname{core}_{G}(K)\right.$.

Given $H, K \in \mathscr{C} \mathfrak{D}(G)$, we use the notation $H \prec K$ to mean that $H<K$ and there is no $R \in \mathcal{C D}(G)$ so that $H<R<K$. If $M$ is the greatest element in $\mathcal{C D}(G)$ and $T$ is the least element in $\mathscr{C D}(G)$ (i.e. $T$ is the Chermak-Delgado subgroup of $G$ ), we say that $A \in \mathcal{C} D(G)$ is an atom if $T \prec A$, and we say that $B \in \mathcal{C D}(G)$ is a coatom if $B \prec M$. Proposition 3 below appears in [6]. The proof in [6] is different than our approach.

Proposition 3. Let $H, K \in \mathcal{C} \mathscr{D}(G)$ with $H \prec K$. Then $H \unlhd K$. And so maximal chains in $\mathcal{C D}(G)$ form subnormal series of $G$. 
Proof. We have $H<K$ and so by Corollary $1, H^{K}<K$, and we know that $H^{K} \in \mathcal{C D}(G)$. Since $H \prec K$, it must be that $H^{K}=H$, i.e. $H \unlhd K$.

Now the greatest element in $\mathcal{C D}(G)$ is normal in $G$ (in fact characteristic in $G$ ), and so maximal chains in $\mathcal{C D}(G)$ form subnormal series of $G$.

Corollary 2. Suppose $G \in \mathcal{C D}(G)$. Then all of the atoms and all of the coatoms of $\mathscr{C D}(G)$ are normal in $G$.

Proof. If $B \in \mathcal{C D}(G)$ is a coatom, then $B \prec G$, and so by Proposition $3, B \unlhd G$. If $A \in \mathcal{C D}(G)$ an atom, then $C_{G}(A) \in \mathcal{C D}(G)$ is a coatom, and so $C_{G}(A) \unlhd G$, and so $C_{G}\left(C_{G}(A)\right)=A \unlhd G$.

The topic of this paper are those groups whose Chermak-Delgado subgroup is the identity subgroup. Consider direct products. Given $H \leq G_{1} \times \cdots \times G_{n}=G$, one sees that $C_{G}(H)=C_{G}\left(\pi_{1}(H) \times \cdots \times \pi_{n}(H)\right)$, where $\pi_{i}$ is the projection map into the $i$-th coordinate. From here one sees that the Chermak-Delgado lattice of a direct product is the Cartesian product of the Chermak-Delgado lattices. Proposition 4 appears in [6]:

Proposition 4. We have $\mathscr{C D}\left(G_{1} \times \cdots \times G_{n}\right)=\mathscr{C D}\left(G_{1}\right) \times \cdots \times \mathcal{C D}\left(G_{n}\right)$.

Corollary 3. We have $1 \in \mathcal{C D}\left(G_{1} \times \cdots \times G_{n}\right)$ if and only if $1 \in \mathscr{C D}\left(G_{i}\right)$ for each $i$.

Proposition 5. Suppose $1 \in \mathcal{C D}(G)$. If $H, K \in \mathcal{C D}(G)$ so that $H \cap K=1$ and $G=H K$, then $G=H \times K$ is a direct product.

Proof. Suppose $H, K \in \mathcal{C D}(G)$ so that $H \cap K=1$ and $G=H K$. Let $A=$ core $_{K}(Z(H))$ and let $B=\operatorname{core}_{A}(K)$. Note that $A$ and $B$ normalize one another, and $A \cap B=1$, and thus $A=\operatorname{core}_{K}(Z(H)) \leq C_{G}\left(\operatorname{core}_{A}(K)\right)=C_{G}(K)^{A}$. So by Proposition $1, A \leq C_{G}(K)$. And since $A \leq Z(H)$ and $G=H K$, we have $A \leq Z(G)=1$. It follows that $1=\operatorname{core}_{K}(Z(H))=\operatorname{core}_{G}(Z(H))$, and so by Proposition $2(2), Z(H)=1$. Now $H \in \mathscr{C D}(G)$ and $m^{*}(G)=|G|$. Hence

$$
|G|=|H|\left|C_{G}(H)\right|=\frac{|H|\left|C_{G}(H)\right|}{|Z(H)|}=\left|H C_{G}(H)\right|,
$$

and so $G=H C_{G}(H)$, and so $H \unlhd G$. By a similar argument, switching $H$ with $K$, one obtains that $K \unlhd G$. And so $G=H \times K$ is a direct product.

How essential is it in Proposition 5 that $1 \in \mathcal{C D}(G)$ ? Can one prove that if $H, K \in \mathcal{C D}(G)$ with $H \cap K=Z(G)$ and $G=H K$, then $H \unlhd G$ and $K \unlhd G$ ? 
The answer is no, and so the property that $1 \in \mathcal{E} \mathfrak{D}(G)$ is needed. We quote [6, Example 1.7]. Let

$$
\begin{aligned}
G=\left\langle a, b, c, d: a^{4}\right. & =b^{2}=c^{2}=d^{2}=[a, b]=[b, c]=[b, d] \\
& =[c, d]=[a, c] b=[a, d] c=1\rangle
\end{aligned}
$$

Then $|G|=32, Z(G)=\langle b\rangle$ has order 2, and $m^{*}(G)=64$. Let $H=\langle a, b\rangle$ and $K=\langle b, d a\rangle$. One can verify that $H, K \in \mathcal{C} D(G), H \cap K=Z(G), G=H K$, but neither $H$ nor $K$ is normal in $G$.

Corollary 4. Suppose $1 \in \mathcal{C D}(G)$. If $G=A B$, where $A$ and $B$ are abelian subgroups of $G$, then $G=1$.

Proof. Since $A$ and $B$ are abelian, $A \cap B \leq Z(G)=1$. So,

$$
|A||B|=|G|=m_{G}(G) \geq m_{G}(A)=|A|\left|C_{G}(A)\right| \geq|A||A|,
$$

so $|B| \geq|A|$. Similarly,

$$
|A||B|=|G|=m_{G}(G) \geq m_{G}(B)=|B|\left|C_{G}(B)\right| \geq|B||B|,
$$

so $|A| \geq|B|$; and it follows that $|A|=|B|$, and we have equalities everywhere in the previous two strings of inequalities. So $A, B \in \mathcal{C} \mathscr{D}(G)$ and so by Proposition $5, G=A \times B$, and so $G$ is abelian, and so $1=Z(G)=G$.

Theorem 1. Suppose $1 \in \mathcal{C} \mathscr{D}(G)$, and suppose $\mathscr{C} \mathscr{D}(G) \cong \mathscr{L}_{1} \times \mathscr{L}_{2}$ for lattices $\mathscr{L}_{1}$ and $\mathscr{L}_{2}$. Then $G=H \times K$ for subgroups $H$ and $K$ with $\mathcal{C D}(H) \cong \mathscr{L}_{1}$ and $\mathcal{C D}(K) \cong \mathscr{L}_{2}$.

Proof. Suppose $1 \in \mathscr{C} \mathscr{D}(G)$ and suppose that $\mathscr{C} \mathscr{D}(G)$ is lattice isomorphic to a Cartesian product $\mathscr{L}_{1} \times \mathscr{L}_{2}$ of lattices $\mathscr{L}_{1}$ and $\mathscr{L}_{2}$. Let $M_{1}$ and $B_{1}$ be the greatest and least elements, respectively, of $\mathscr{L}_{1}$ and let $M_{2}$ and $B_{2}$ be the greatest and least elements, respectively, of $\mathscr{L}_{2}$. Note that $\left(B_{1}, M_{2}\right) \vee\left(M_{1}, B_{2}\right)=\left(M_{1}, M_{2}\right)$ is the greatest element of $\mathscr{L}_{1} \times \mathscr{L}_{2}$ and $\left(B_{1}, M_{2}\right) \wedge\left(M_{1}, B_{2}\right)=\left(B_{1}, B_{2}\right)$ is the least element of $\mathscr{L}_{1} \times \mathscr{L}_{2}$. And since $\mathscr{C D}(G)$ is lattice isomorphic to $\mathscr{L}_{1} \times \mathscr{L}_{2}$, there is $H, K \in \mathscr{C} \mathscr{D}(G)$ corresponding to $\left(M_{1}, B_{2}\right),\left(B_{1}, M_{2}\right)$, respectively, in $\mathscr{L}_{1} \times \mathscr{L}_{2}$ so that $H \cap K=1$ and $H K=G$. By Proposition $5, G=H \times K$ is a direct product. By Proposition 4, $\mathcal{C D}(G)=\mathscr{C} \mathscr{D}(H) \times \mathcal{C} \mathscr{D}(K)$. And so we have a lattice isomorphism between $\mathscr{C D}(H) \times \mathscr{C} \mathscr{D}(K)$ and $\mathscr{L}_{1} \times \mathscr{L}_{2}$, where $H$ corresponds to $\left(M_{1}, B_{2}\right)$ and $K$ corresponds to $\left(B_{1}, M_{2}\right)$. Thus $\mathcal{C D}(H) \cong \mathscr{L}_{1}$ and $\mathcal{C D}(K) \cong \mathscr{L}_{2}$.

By induction, this theorem extends to a direct product/Cartesian product of $n$ number of groups/CD lattices. 
A group $G$ is said to be indecomposable if $G$ cannot be written as an (internal) direct product $H \times K$ with $H \neq 1$ and $K \neq 1$. A lattice $\mathscr{L}$ is said to be indecomposable if $\mathscr{L}$ is not lattice isomorphic to a Cartesian product $\mathscr{L}_{1} \times \mathscr{L}_{2}$ of lattices with $\mathscr{L}_{1}$ and $\mathscr{L}_{2}$ both nontrivial (a trivial lattice is a lattice consisting of a single point).

Corollary 5. Suppose $1 \in \mathcal{C D}(G)$. Then $G$ is indecomposable if and only if $\mathcal{C D}(G)$ is indecomposable.

Proof. Suppose $1 \in \mathscr{C D}(G)$ and suppose that $\mathscr{C D}(G)$ is indecomposable. If $G=H \times K$, then by Proposition $4, \mathcal{C D}(G)=\mathscr{C D}(H) \times \mathscr{C D}(K)$. Since $\mathcal{C D}(G)$ is indecomposable, at least one of $\mathcal{C D}(H)$ or $\mathcal{C D}(K)$ is trivial, say without loss of generality that $\mathcal{C D}(H)$ is trivial. By Corollary 3 , since $1 \in \mathcal{C D}(G), 1 \in \mathcal{C D}(H)$, and so $\mathscr{C D}(H)=\{1\}$. And so $H=1$, and thus $G$ is indecomposable.

Suppose $1 \in \mathscr{C D}(G)$ and suppose $G$ is indecomposable. Suppose that $\mathscr{C D}(G)$ is lattice isomorphic to a Cartesian product $\mathscr{L}_{1} \times \mathscr{L}_{2}$ of lattices $\mathscr{L}_{1}$ and $\mathscr{L}_{2}$. By Theorem 1, we have $G=H \times K$ for subgroups $H$ and $K$ with $\mathscr{C D}(H) \cong \mathscr{L}_{1}$ and $\mathcal{C D}(K) \cong \mathscr{L}_{2}$. And since $G$ is indecomposable, at least one of $H$ or $K$ is trivial, and so at least one of $\mathscr{L}_{1}$ or $\mathscr{L}_{2}$ is trivial, and so $\mathscr{C}(G)$ is indecomposable.

In [11], the author studied CD-simple groups, which are groups, $G$, having the property that $\mathscr{C D}(G)=\{1, G\}$. We define a group, $G$, to be CD-minimal if $1 \in \mathcal{C D}(G)$ and $G$ is indecomposable. So every CD-simple group is CD-minimal, but not vice versa.

Proposition 6. Suppose $G$ is $C D$-minimal but not $C D$-simple. If $A \in \mathcal{C D}(G)$ is an atom and if $B \in \mathcal{C D}(G)$ is a coatom, then $A \leq B$.

Proof. By Corollary 2, both $1 \neq A$ and $1 \neq B$ are normal in $G$. If $A \cap B=1$, then $G=A \times B$ is a direct product, contrary to the assumption that $G$ is indecomposable. So $A \cap B \neq 1$, and since $A$ is an atom in $\mathcal{C D}(G), A \leq B$.

Proposition 7. Suppose $G$ is $C D$-minimal but not $C D$-simple. If $A \in \mathcal{C D}(G)$ is an atom, then $A$ is abelian, $A \unlhd G$, and $|A|$ contains primes $p \neq q$.

Proof. Let $1 \neq A \in \mathcal{C D}(G)$ be an atom. Then $C_{G}(A) \in \mathcal{C D}(G)$ is a coatom, and by Proposition 6, $A \leq C_{G}(A)$, i.e. $A$ is abelian. By Corollary $2, A \unlhd G$. Let $N \leq A$ be a minimal normal subgroup of $G$. So $|N|=p^{k}$ for some prime $p$ and some $k$. Now, as $N$ is minimal normal, $G / C_{G}(N)$ acts faithfully and irreducibly on $N$, and so $\left|G / C_{G}(N)\right|$ is not a power of $p$. And so a prime $q \neq p$ divides $\left|G / C_{G}(N)\right|$. Now $\left|G / C_{G}(N)\right|$ divides $\left|G / C_{G}(A)\right|$, and since $A \in \mathcal{C D}(G)$, we have $|A|=\left|G / C_{G}(A)\right|$. Thus $|A|$ contains primes $p \neq q$. 
Lemma 1. If $H, K \in \mathcal{C D}(G)$ and $K \leq H$, then $|H: K|=\left|C_{G}(K): C_{G}(H)\right|$.

Proof. As $H, K \in \mathcal{C} \mathscr{D}(G)$, it follows that $|H|\left|C_{G}(H)\right|=|K|\left|C_{G}(K)\right|$, and so $|H: K|=\left|C_{G}(K): C_{G}(H)\right|$.

Corollary 6 follows immediately:

Corollary 6. Suppose $G$ is $C D$-minimal but not $C D$-simple. If $B \in \mathcal{C} \mathscr{D}(G)$ is a coatom, then $C_{G}(B) \leq B, B \unlhd G$, and $|G: B|$ contains primes $p \neq q$

Corollary 7. Suppose $1 \in \mathcal{C} \mathscr{D}(G)$. If $1 \neq H \in \mathcal{C} \mathscr{D}(G)$, then $H$ is not a $p$-group for any prime $p$.

Proof. If we prove Corollary 7 for any CD-minimal group, then by Corollary 3 , the result will follow for any arbitrary group $X$ with $1 \in \mathcal{C} \mathscr{D}(X)$. So suppose $G$ is a CD-minimal group. If $G$ is CD-simple, then $G$ is not a $p$-group for any prime $p$ since $Z(G)=1$. If $G$ is CD-minimal and not CD-simple, and $1 \neq H \in \mathcal{C} \mathscr{D}(G)$, then $H$ contains an atom $A \in \mathcal{C} \mathscr{D}(G)$, and so by Proposition 7, $H$ is not a $p$-group for any prime $p$.

Corollary 8. Suppose $1 \in \mathscr{C} D(G)$. If $G \neq H \in \mathscr{C} \mathscr{D}(G)$, then $|G: H|$ contains at least two different primes, and it follows that $H$ is not a maximal subgroup of $G$.

Proof. If we prove Corollary 8 for any CD-minimal group, then by Corollary 3, the result will follow for any arbitrary group $X$ with $1 \in \mathcal{C} \mathscr{D}(X)$. So suppose $G$ is a CD-minimal group. If $G$ is CD-simple, then $1=Z(G)=H \neq G$ implies that $|G|=|G: 1|$ contains at least two different primes. So suppose that $G$ is CD-minimal and not CD-simple. Suppose $G \neq H \in \mathcal{C} D(G)$. Then we have $H \leq B$, where $B \in \mathscr{C} \mathscr{D}(G)$ is a coatom. Note that $|G: B|$ divides $|G: H|$, and by Corollary $6,|G: B|$ contains primes $p \neq q$, and so $|G: H|$ contains primes $p \neq q$. Suppose by way of contradiction that $H$ is a maximal subgroup of $G$ and let $P$ be a Sylow $p$-subgroup of $G$. Then $H<H P$. Since $H$ is maximal in $G, H P=G$. But $|G: H P|$ contains a prime $q$, a contradiction.

Denote the class of all finite groups with a trivial Chermak-Delgado subgroup by $\mathcal{T}$. The reader summarizes results as they pertain to $\mathcal{T}$. The class $\mathcal{T}$ non-trivially intersects the class of solvable groups, as the symmetric group $S_{4}$ is CD-simple. And the class $\mathcal{T}$ properly contains the class of non-abelian simple groups. All of the examples of $\mathrm{CD}$-minimal groups in the next section are solvable groups.

Given $G \in \mathcal{T}, \mathcal{C D}(G)$ does not contain any non-trivial $p$-groups by Corollary 7. It was shown in [11] (see Proposition 6 there) that given $G \in \mathcal{T}, \mathcal{C} \mathscr{D}(G)$ 
does not contain any non-trivial, cyclic subgroups that are normal in $G$. We can apply Proposition 2(2) to show that given $G \in \mathcal{T}, \mathcal{C D}(G)$ does not contain any non-trivial, cyclic groups.

Let $\mathcal{T}^{*}$ denote the class of all lattices $\mathcal{C D}(G)$ with $G \in \mathcal{T}$. The class $\mathcal{T}^{*}$ is closed under Cartesian products by Corollary 4 , and if $\mathscr{L} \in \mathcal{T}^{*}$ is lattice isomorphic to a Cartesian product of lattices, then each lattice appearing in that Cartesian product is in $\mathcal{T}^{*}$; this is by Theorem 1 . Given indecomposable $\mathscr{L} \in \mathcal{T}^{*}$ with $|\mathscr{L}|>2$, and given $X \in \mathscr{L}$ an atom, and $Y \in \mathscr{L}$ a coatom, we have that $X \leq Y$ by Proposition 6. A lattice $\mathscr{L}$ is said to be a quasi-antichain of width $n$ if $\mathscr{L}$ contains exactly $n$ atoms, and every atom in $\mathscr{L}$ is a coatom in $\mathscr{L}$. We denote a quasiantichain lattice of width $n$ by $\mathcal{M}_{n}$. And so given indecomposable $\mathscr{L} \in \mathcal{T}^{*}, \mathscr{L}$ is not isomorphic to $\mathcal{M}_{n}$ for any $n>1$. Note that the group $1=G \in \mathcal{T}$ has that $\mathcal{C D}(G)=\{1\} \cong \mathcal{M}_{0}$, and in the next section we construct a group $G \in \mathcal{T}$ so that $\mathcal{C D}(G) \cong \mathcal{M}_{1}$, but beyond that, $\mathcal{C D}(G)$ is never a quasi-antichain for indecomposable $G \in \mathcal{T}$. This is surprising considering the prevalence of $\mathcal{M}_{n}$ lattices in the theory of Chermak-Delgado $p$-group lattices, see [5] and [2]. We will see in the next section that quasi-antichain $p$-group lattices do play a major role in the CD lattices of some CD-minimal group examples.

Suppose that $\mathscr{L}$ is a lattice with greatest element $M$ and least element $B$. Given $X \in \mathscr{L}$, we say that $Y \in \mathscr{L}$ is a complement of $X$ if $X \vee Y=M$ and $X \wedge Y=B$. Given indecomposable $\mathscr{L} \in \mathcal{T}^{*}$, it follows from Proposition 5 that the only elements in $\mathscr{L}$ that have complements in $\mathscr{L}$ are the greatest and least elements in $\mathscr{L}$. This further restricts the structure of indecomposable $\mathscr{L} \in \mathcal{T}^{*}$.

\section{Examples of CD-Minimal Groups which are not CD-Simple}

Do there exist CD-minimal groups which are not CD-simple? The answer is yes!

In this section, $\mathbb{Z}_{n}$ denotes the group of integers modulo $n, C_{n}$ denotes the cyclic group of order $n, S_{n}$ denotes the symmetric group on $n$ elements, $Q_{8}$ denotes the quaternion group of order 8 , and $Q D_{16}$ denotes the quasidihedral group of order 16. Note that $Q D_{16}=\left\langle r, s: r^{8}=s^{2}=1, s r s=r^{3}\right\rangle$. Given a group action of a group $T$ on a group $N$, we let $[N] T$ denote the semidirect product.

Lemma 2. Suppose $1 \neq P$ is a $p$-group and $T$ is a group so that $T$ acts on $P$ and so that the restricted action of $T$ on $Z(P)$ is faithful and irreducible, and let $G=[P] T$. If $1 \neq A \unlhd G$ and $A$ is abelian, then $Z(P) \leq A \leq C_{G}(A) \leq P$.

Proof. Note that if $A \cap Z(P)=1$, then since $A \unlhd G$, and $Z(P) \unlhd G$, we have $A \leq C_{G}(Z(P))=P$, since $T$ acts faithfully on $Z(P)$. But since $1 \neq A \unlhd P$ and $1 \neq P$ is a $p$-group, we have $A \cap Z(P)>1$, a contradiction. So $A \cap Z(P)>1$. 
Now, since $T$ acts irreducibly on $Z(P)$, it follows that $Z(P) \leq A$. And so we have $A \leq C_{G}(A) \leq C_{G}(Z(P))=P$.

For $i \in\{1,2\}$, let $P_{i}$ be a 2-group so that

- $P_{i} \in \mathcal{C D}\left(P_{i}\right)$,

- $Z\left(P_{i}\right) \cong \mathbb{Z}_{2} \times \mathbb{Z}_{2}$,

- there exists a group $T_{i}=\left[H_{i}\right] K_{i}$ with $\left|H_{i}\right|=3$ and $\left|K_{i}\right|=2$ so that $T_{i}$ acts on $P_{i}$ and so that the restricted action of $T_{i}$ on $Z\left(P_{i}\right)$ is faithful and irreducible (and so $\left.T_{i} \cong \operatorname{Aut}\left(\mathbb{Z}_{2} \times \mathbb{Z}_{2}\right) \cong S_{3}\right)$.

Let $P_{3}$ be a 3 -group so that $P_{3} \in \mathcal{C} \mathscr{D}\left(P_{3}\right)$, so that $Z\left(P_{3}\right) \cong \mathbb{Z}_{3} \times \mathbb{Z}_{3}$, and so that there exists a group $T_{3}=\left[H_{3}\right] K_{3}$ with $\left|H_{3}\right|=8$ and $\left|K_{3}\right|=2$ so that $T_{3}$ acts on $P_{3}$ and so that the restricted action of $T_{3}$ on $Z\left(P_{3}\right)$ is faithful and irreducible (note that a Sylow 2-subgroup of $\operatorname{Aut}\left(\mathbb{Z}_{3} \times \mathbb{Z}_{3}\right)$ is isomorphic to $Q D_{16}=[\langle r\rangle]\langle s\rangle$.)

Let $G_{1}=\left[P_{1}\right] T_{1}, G_{2}=\left[P_{2}\right] T_{2}, G_{3}=\left[P_{3}\right] T_{3}$, and let $G=G_{1} \times G_{2} \times G_{3}$.

Let $\pi_{i}$ denote the projection homomorphism of $G$ onto the $i$ th coordinate. Let $S \leq G$ so that for each $i \in\{1,2,3\}$, one has $\pi_{i}(S)=G_{i}, S \cap G_{i}=\left[P_{i}\right] H_{i}$, and $S \cap\left(K_{1} \times K_{2} \times K_{3}\right)$ is the diagonal subgroup of $K_{1} \times K_{2} \times K_{3}$ (and so $\left|S \cap\left(K_{1} \times K_{2} \times K_{3}\right)\right|=2$. $)$

Lemma 3. Let $S$ be a group constructed as above. If $H \in \mathcal{C D}(S)$, and if for each $i \in\{1,2,3\}$,

$$
Z\left(P_{i}\right) \leq \pi_{i}(H) \leq P_{i}
$$

then $H, C_{S}(H) \in\left(\mathscr{C D}\left(P_{1}\right) \times \mathscr{C} \mathscr{D}\left(P_{2}\right) \times \mathcal{C D}\left(P_{3}\right)\right)$.

Proof. Note that

$$
\begin{aligned}
C_{S}(H) & =C_{S}\left(\pi_{1}(H) \times \pi_{2}(H) \times \pi_{3}(H)\right) \\
& \leq C_{S}\left(Z\left(P_{1}\right) \times Z\left(P_{2}\right) \times Z\left(P_{3}\right)\right)=P_{1} \times P_{2} \times P_{3}
\end{aligned}
$$

since each $T_{i}$ acts faithfully on $Z\left(P_{i}\right)$. And so

$$
C_{S}(H)=C_{P_{1}}\left(\pi_{1}(H)\right) \times C_{P_{2}}\left(\pi_{2}(H)\right) \times C_{P_{3}}\left(\pi_{3}(H)\right) .
$$

And since $H \in \mathscr{C} \mathscr{D}(S)$, it follows that $H=\pi_{1}(H) \times \pi_{2}(H) \times \pi_{3}(H)$. And so $H, C_{S}(H) \in\left(\mathcal{C D}\left(P_{1}\right) \times \mathscr{C} \mathscr{D}\left(P_{2}\right) \times \mathscr{C} \mathscr{D}\left(P_{3}\right)\right)$.

Theorem 2 below is an example of a CD lattice extension theorem. There have been papers written about CD lattice extension theorems for $p$-groups, see [3,4]. This is the first non $p$-group CD lattice extension theorem that the author is aware of. 
Theorem 2. Let $S$ be a group constructed as above, and suppose $\left|P_{1}\right|=2^{a}$, $\left|P_{2}\right|=2^{b}$, and $\left|P_{3}\right|=3^{c}$ for some $a, b, c$. Then $S$ is a CD-minimal group of order $2^{a+b+4} \cdot 3^{c+2}$ and $\mathscr{C D}(S)=\left(\mathscr{C D}\left(P_{1}\right) \times \mathscr{C} \mathfrak{D}\left(P_{2}\right) \times \mathscr{C} \mathfrak{D}\left(P_{3}\right)\right) \cup\{1, S\}$.

Proof. Let $S$ be a group constructed as above, and suppose $\left|P_{1}\right|=2^{a},\left|P_{2}\right|=2^{b}$, and $\left|P_{3}\right|=3^{c}$ for some $a, b, c$. And so we have $\left|Z\left(P_{1}\right)\right|=4,\left|Z\left(P_{2}\right)\right|=4$, and $\left|Z\left(P_{3}\right)\right|=9$. And since $P_{1} \in \mathcal{C} \mathscr{D}\left(P_{1}\right), P_{2} \in \mathcal{C} \mathscr{D}\left(P_{2}\right)$, and $P_{3} \in \mathcal{C} \mathscr{D}\left(P_{3}\right)$, we have that

$$
\begin{aligned}
2^{a+b+4} \cdot 3^{c+2} & =m^{*}\left(P_{1}\right) \cdot m^{*}\left(P_{2}\right) \cdot m^{*}\left(P_{3}\right) \\
& =|S|=\left|P_{1}\right|\left|H_{1}\right|\left|P_{2}\right|\left|H_{2}\right|\left|P_{3}\right|\left|H_{3}\right| 2 .
\end{aligned}
$$

We now establish that the Chermak-Delgado subgroup of $S$ is 1, and along the way we determine all of the abelian, normal subgroups of $S$ that are in $\mathscr{C D}(S)$. Note that $m_{S}(1)=|S|$. We know that the Chermak-Delgado subgroup of $S$ is a characteristic, abelian subgroup of $S$, and so let us suppose that $1 \neq A \unlhd S$ with $A$ abelian and $A \in \mathcal{C} \mathscr{D}(S)$. Since $A \unlhd S$, for each $i \in\{1,2,3\}$, it follows that $\pi_{i}(A) \unlhd \pi_{i}(S)=G_{i}$. By Lemma 2 , if $\pi_{i}(A) \neq 1$, then

$$
Z\left(P_{i}\right) \leq \pi_{i}(A) \leq C_{G_{i}}\left(\pi_{i}(A)\right) \leq P_{i} .
$$

And so $C_{G_{i}}\left(\pi_{i}(A)\right)=\pi_{i}\left(C_{S}(A)\right)$ and $\left|\pi_{i}(A) \| \pi_{i}\left(C_{S}(A)\right)\right| \leq m^{*}\left(P_{i}\right)$. We now show that for each $i \in\{1,2,3\}, \pi_{i}(A) \neq 1$. Suppose not. Then there is $j_{0} \in\{1,2,3\}$ so that $\pi_{j_{0}}(A)=1$. And since $1 \neq A$, there is $i_{0} \in\{1,2,3\}$ so that $\pi_{i_{0}}(A) \neq 1$. So if $\pi_{j}(A)=1$, then $\pi_{j}\left(C_{S}(A)\right)=\left[P_{j}\right] H_{j}$. This is true because $T_{i_{0}}$ acts faithfully on $Z\left(P_{i_{0}}\right) \leq \pi_{i_{0}}(A)$, and so the diagonal subgroup of $K_{1} \times K_{2} \times K_{3}$ will not centralize $A$. Thus, if $\pi_{j}(A)=1$, then

$$
\left|\pi_{j}\left(C_{S}(A)\right)\right|=\left|P_{j}\right|\left|H_{j}\right|<\left|P_{j}\right|\left|Z\left(P_{j}\right)\right|=m^{*}\left(P_{j}\right) .
$$

And so

$$
\begin{aligned}
m_{S}(A) & =|A|\left|C_{S}(A)\right| \\
& \leq\left|\pi_{1}(A)\right|\left|\pi_{2}(A)\left\|\pi_{3}(A)\right\| \pi_{1}\left(C_{S}(A)\right)\right|\left|\pi_{2}\left(C_{S}(A)\right)\right|\left|\pi_{3}\left(C_{S}(A)\right)\right| \\
& <m^{*}\left(P_{1}\right) \cdot m^{*}\left(P_{2}\right) \cdot m^{*}\left(P_{3}\right)=|S|
\end{aligned}
$$

which contradicts $A \in \mathscr{C} \mathscr{D}(S)$.

So for each $i \in\{1,2,3\}, \pi_{i}(A) \neq 1$. By Lemma $2, Z\left(P_{i}\right) \leq \pi_{i}(A) \leq P_{i}$ for each $i \in\{1,2,3\}$. So by Lemma $3, A, C_{S}(A) \in\left(\mathcal{C D}\left(P_{1}\right) \times \mathcal{C D}\left(P_{2}\right) \times \mathcal{C} \mathscr{D}\left(P_{3}\right)\right)$. Thus $m_{S}(A)=m^{*}\left(P_{1}\right) \cdot m^{*}\left(P_{2}\right) \cdot m^{*}\left(P_{3}\right)=|S|$. Hence we have shown that the Chermak-Delgado subgroup of $S$ is the identity, and hence $m^{*}(S)=|S|$, and so

$$
\left(\mathscr{C D}\left(P_{1}\right) \times \mathscr{C} \mathscr{D}\left(P_{2}\right) \times \mathcal{C D}\left(P_{3}\right)\right) \cup\{1, S\} \subseteq \mathscr{C D}(S) .
$$


Furthermore, we know that if $1 \neq A \unlhd S$ with $A$ abelian and $A \in \mathcal{C} \mathscr{D}(S)$, then $A \in\left(\mathscr{C D}\left(P_{1}\right) \times \mathscr{C} \mathscr{D}\left(P_{2}\right) \times \mathscr{C} \mathfrak{D}\left(P_{3}\right)\right)$.

Note that $Z\left(P_{1}\right) \times Z\left(P_{2}\right) \times Z\left(P_{3}\right)$ is an atom in $\mathcal{C D}(S)$. This is true because otherwise, $Z\left(P_{1}\right) \times Z\left(P_{2}\right) \times Z\left(P_{3}\right)$ would properly contain an atom $1 \neq A$ of $\mathcal{C D}(S)$. By Corollary $2, A \unlhd S$. But then $A \in\left(\mathscr{C D}\left(P_{1}\right) \times \mathscr{C} D\left(P_{2}\right) \times \mathscr{C} \mathscr{D}\left(P_{3}\right)\right)$, a contradiction. We now show that $Z\left(P_{1}\right) \times Z\left(P_{2}\right) \times Z\left(P_{3}\right)$ is the unique atom in $\mathcal{C D}(S)$. Suppose not. So $1 \neq N$ is another atom in $\mathcal{C D}(S)$. So by Corollary 2 , we have $N \unlhd S$. Since $N \cap\left(Z\left(P_{1}\right) \times Z\left(P_{2}\right) \times Z\left(P_{3}\right)\right)=1$, it follows that $N \leq C_{S}\left(Z\left(P_{1}\right) \times Z\left(P_{2}\right) \times Z\left(P_{3}\right)\right)=P_{1} \times P_{2} \times P_{3}$. And since $P_{1} \times P_{2} \times P_{3}$ is nilpotent, we have that $N \cap\left(Z\left(P_{1}\right) \times Z\left(P_{2}\right) \times Z\left(P_{3}\right)\right)>1$, a contradiction. So $Z\left(P_{1}\right) \times Z\left(P_{2}\right) \times Z\left(P_{3}\right)$ is the unique atom in $\mathcal{C D}(S)$.

If $H \in \mathcal{C} \mathscr{D}(S)$ with $H \neq 1$ and $H \neq S$, then

$$
Z\left(P_{1}\right) \times Z\left(P_{2}\right) \times Z\left(P_{3}\right) \leq H, C_{S}(H),
$$

and so

$$
C_{S}(H), H \leq P_{1} \times P_{2} \times P_{3},
$$

and so by Lemma $3, H \in\left(\mathscr{C D}\left(P_{1}\right) \times \mathscr{C} \mathscr{D}\left(P_{2}\right) \times \mathscr{C} \mathscr{D}\left(P_{3}\right)\right)$. Thus we have that $\mathscr{C D}(S)=\left(\mathcal{C D}\left(P_{1}\right) \times \mathcal{C} \mathscr{D}\left(P_{2}\right) \times \mathscr{C} \mathscr{D}\left(P_{3}\right)\right) \cup\{1, S\}$.

Finally, since $\mathscr{C D}(S)$ contains a unique atom, it follows that $\mathscr{C D}(S)$ is an indecomposable lattice. And so by Corollary $5, S$ is indecomposable. Thus, $S$ is a CD-minimal group with the prescribed properties.

We desire examples of groups $P_{1}, P_{2}, P_{3}$ and acting groups $T_{1}, T_{2}, T_{3}$ in Theorem 2. One can take $P_{1}=\mathbb{Z}_{2} \times \mathbb{Z}_{2}, P_{2}=\mathbb{Z}_{2} \times \mathbb{Z}_{2}, P_{3}=\mathbb{Z}_{3} \times \mathbb{Z}_{3}$, and $T_{1}=\operatorname{Aut}\left(\mathbb{Z}_{2} \times \mathbb{Z}_{2}\right), T_{2}=\operatorname{Aut}\left(\mathbb{Z}_{2} \times \mathbb{Z}_{2}\right)$, and $T_{3}$ to be a Sylow 2-subgroup of $\operatorname{Aut}\left(\mathbb{Z}_{3} \times \mathbb{Z}_{3}\right)$. Let $S_{0}$ be the CD-minimal group constructed using each of these examples. The author thanks Peter Hauck for providing this construction. The group $S_{0}$ has order $2^{8} \cdot 3^{4}=20736$. We have $\mathcal{C D}\left(S_{0}\right) \cong \mathcal{M}_{1}$. Note that the unique atom/coatom, $A=\left(\mathbb{Z}_{2} \times \mathbb{Z}_{2}\right) \times\left(\mathbb{Z}_{2} \times \mathbb{Z}_{2}\right) \times\left(\mathbb{Z}_{3} \times \mathbb{Z}_{3}\right)$, in $\mathcal{C D}\left(S_{0}\right)$ is abelian, $A \unlhd S_{0}$, and $|A|$ contains at least two primes. This (thankfully) agrees with the theory established in Section 1.

Proposition 8 below appears in [5]. The proof makes use of [9, Exercise 39].

Proposition 8. Let $p$ be a prime and $n$ a positive integer. Let $P$ be the group of all $3 \times 3$ lower triangular matrices over $\mathrm{GF}\left(p^{n}\right)$ with ones along the diagonal. The Chermak-Delgado lattice of $P$ is a quasi-antichain of width $p^{n}+1$ and all subgroups in the middle antichain are abelian.

Proposition 9. Let $p$ be a prime and $n$ a positive integer. Let $P$ be the group of all $3 \times 3$ lower triangular matrices over $\mathrm{GF}\left(p^{n}\right)$ with ones along the diagonal. Then 
there exists $T \leq \operatorname{Aut}(P)$ so that $T=[H] K$ with $|H|=p^{n}-1$ and $|K|=n$, and so that the restricted action of $T$ on $Z(P) \cong \mathbb{Z}_{p} \times \cdots \times \mathbb{Z}_{p}$ is faithful and irreducible. $n$ times

Proof. Note that

$$
\left[\begin{array}{ccc}
1 & 0 & 0 \\
a_{1} & 1 & 0 \\
b_{1} & c_{1} & 1
\end{array}\right] \cdot\left[\begin{array}{ccc}
1 & 0 & 0 \\
a_{2} & 1 & 0 \\
b_{2} & c_{2} & 1
\end{array}\right]=\left[\begin{array}{ccc}
1 & 0 & 0 \\
a_{1}+a_{2} & 1 & 0 \\
b_{1}+b_{2}+c_{1} a_{2} & c_{1}+c_{2} & 1
\end{array}\right],
$$

and note that

$$
Z(P)=\left\{\left[\begin{array}{lll}
1 & 0 & 0 \\
0 & 1 & 0 \\
b & 0 & 1
\end{array}\right]: b \in \mathrm{GF}\left(p^{n}\right)\right\} \cong \begin{aligned}
& p \times \cdots \times \mathbb{Z}_{p} . \\
& n \text { times }
\end{aligned}
$$

Let $x$ be a generator of the group of units of $\operatorname{GF}\left(p^{n}\right)$. Define

$$
\left(\left[\begin{array}{lll}
1 & 0 & 0 \\
a & 1 & 0 \\
b & c & 1
\end{array}\right]\right)^{r}=\left[\begin{array}{ccc}
1 & 0 & 0 \\
x \cdot a & 1 & 0 \\
x \cdot b & c & 1
\end{array}\right]
$$

Note that $r \in \operatorname{Aut}(P)$ and $H=\langle r\rangle$ has order $p^{n}-1$. Define

$$
\left(\left[\begin{array}{lll}
1 & 0 & 0 \\
a & 1 & 0 \\
b & c & 1
\end{array}\right]\right)=\left[\begin{array}{ccc}
1 & 0 & 0 \\
a^{p} & 1 & 0 \\
b^{p} & c^{p} & 1
\end{array}\right]
$$

Note that $s \in \operatorname{Aut}(P)$ and $K=\langle s\rangle$ has order $n$, and observe that $s^{-1} r s=r^{p}$, and $T=[H] K \leq \operatorname{Aut}(P)$ and acts faithfully and irreducibly on $Z(P)$.

Proposition 9 with $p=2$ and $n=2$ yields examples of groups $P_{1}, P_{2}$ and $T_{1}, T_{2}$ in Theorem 2. Proposition 9 with $p=3$ and $n=2$ yields examples of groups $P_{3}$ and $T_{3}$ in Theorem 2. Proposition 8 tells us that each $P_{i} \in \mathcal{C D}\left(P_{i}\right)$ and tells us the structure of $\mathcal{C D}\left(P_{i}\right)$.

The following example was provided by Ben Brewster, and yields examples of groups $P_{1}, P_{2}$ and $T_{1}, T_{2}$ in Theorem 2. Let $D=\left\{(x, x, x): x \in Z\left(Q_{8}\right)\right\}$, and let $P=\left(Q_{8} \times Q_{8} \times Q_{8}\right) / D$. Consider the natural action of $T=S_{3}$ on $P$ via permutation of the coordinates. Then $T$ acts faithfully and irreducibly on $Z(P) \cong \mathbb{Z}_{2} \times \mathbb{Z}_{2}$. It can be shown that

$$
\mathscr{C D}(P)=\left\{(X \times Y \times Z) / D: X, Y, Z \in \mathcal{C D}\left(Q_{8}\right)\right\} .
$$


The author verified this through use of GAP. We know that $\mathcal{C D}\left(Q_{8}\right)$ consists of all of the subgroups of $Q_{8}$ that contain $Z\left(Q_{8}\right)$, and so $\mathcal{C D}\left(Q_{8}\right)$ is a quasi-antichain of width 3. And so $\mathscr{C D}(P) \cong \mathcal{M}_{3} \times \mathcal{M}_{3} \times \mathcal{M}_{3}$. The author would like to remark that $Q_{8}$ is an example of an extraspecial $p$-group, and it is true that for any extraspecial $p$-group, $R, \mathcal{C D}(R)$ consists of all of the subgroups of $R$ that contain $Z(R)$. This was mentioned in [8, Example 2.8]; the result follows from considering a nondegenerate bilinear form induced by commutation that is endowed upon $R / Z(R)$ when viewed as a vector space over $\mathbb{F}_{p}$. The author would like to remark that in a recent paper, [12], the groups $G$ having the property that $\mathcal{C D}(G)$ consists of all of the subgroups of $G$ that contain $Z(G)$ are classified.

We encourage the reader to collect all of the above examples that work in Theorem 2. We arrive at twelve examples of CD-minimal groups that are not $\mathrm{CD}$-simple, with each example having a different CD lattice. These lattices are extensions of Cartesian products of quasi-antichain lattices by a top and bottom element.

We end with some open questions. Are there examples of CD-minimal groups, not CD-simple, that are $\{p, q\}$-groups for $\{p, q\} \neq\{2,3\}$ ? Obviously, one desires more theory on the class of groups $\mathcal{T}$ and the class of lattices $\mathcal{T}^{*}$, and more examples of groups and lattices in each class would further that theory. Is the appearance of quasi-antichain $p$-group lattices in this construction mere coincidence, perhaps because of the small order of groups involved in the construction, or is there a deeper reason that quasi-antichain lattices appear prevalently in CD lattices? For groups in $\mathcal{T}$ and lattices in $\mathcal{T}^{*}$, we have established a relationship between direct products of groups and Cartesian products of lattices. Are there more relationships between lattice theoretic properties of $\mathcal{C D}(G)$ and group theoretic properties of $G$ ?

Acknowledgments. The author thanks the referee for valuable comments and for providing a short proof of Proposition 1.

\section{Bibliography}

[1] B. Amberg, S. Franciosi and F. de Giovanni, Products of Groups, Oxford Math. Monogr., Clarendon Press, New York, 1992.

[2] L. An, Groups whose Chermak-Delgado lattice is a quasi-antichain, preprint (2017), https://arxiv.org/abs/1705.06456.

[3] L. An, J. P. Brennan, H. Qu and E. Wilcox, Chermak-Delgado lattice extension theorems, Comm. Algebra 43 (2015), no. 5, 2201-2213.

[4] B. Brewster, P. Hauck and E. Wilcox, Groups whose Chermak-Delgado lattice is a chain, J. Group Theory 17 (2014), no. 2, 253-265. 
[5] B. Brewster, P. Hauck and E. Wilcox, Quasi-antichain Chermak-Delgado lattices of finite groups, Arch. Math. (Basel) 103 (2014), no. 4, 301-311.

[6] B. Brewster and E. Wilcox, Some groups with computable Chermak-Delgado lattices, Bull. Aust. Math. Soc. 86 (2012), no. 1, 29-40.

[7] A. Chermak and A. Delgado, A measuring argument for finite groups, Proc. Amer. Math. Soc. 107 (1989), no. 4, 907-914.

[8] G. Glauberman, Centrally large subgroups of finite p-groups, J. Algebra $\mathbf{3 0 0}$ (2006), no. $2,480-508$.

[9] B. Huppert, Endliche Gruppen. I, Grundlehren Math. Wiss. 134, Springer, Berlin, 1967.

[10] I. M. Isaacs, Finite Group Theory, Grad. Stud. Math. 92, American Mathematical Society, Providence, 2008.

[11] R. McCulloch, Chermak-Delgado simple groups, Comm. Algebra 45 (2017), no. 3, 983-991.

[12] M. Tărnăuceanu, A note on the Chermak-Delgado lattice of a finite group, Comm. Algebra (2017), DOI 10.1080/00927872.2017.1355374.

Received August 10, 2017; revised October 24, 2017.

\section{Author information}

Ryan McCulloch, Department of Mathematics, University of Bridgeport,

Bridgeport, CT 06604, USA.

E-mail: rmccullo@bridgeport.edu 